\title{
Landing Impact Intensities for Jumping Exercises From the OPTIMA-Ex Trial in Trained and Untrained Women
}

\author{
Conor Lambert, ${ }^{1,2}$ Belinda R. Beck, ${ }^{1,2,3}$ and Benjamin K. Weeks ${ }^{1,2}$ \\ ${ }^{1}$ Menzies Health Institute Queensland, Griffith University, Gold Coast, Queensland, Australia; ${ }^{2}$ School of Allied Health Sciences, Griffith \\ University, Gold Coast, Queensland, Australia; and ${ }^{3}$ The Bone Clinic, Brisbane, Queensland, Australia
}

\begin{abstract}
Lambert, C, Beck, BR, and Weeks, BK. Landing impact intensities for jumping exercises from the OPTIMA-Ex trial in trained and untrained women. J Strength Cond Res $X X(X)$ : 000-000, 2019-High-intensity mechanical loads are required to elicit a positive adaptive bone response. Our aim was to quantify the mechanical loads of impact exercises used in each progressive stage of a bone-targeted exercise intervention (the OPTIMA-Ex trial) and to investigate differences in mechanical loads between untrained and trained subjects. A randomized repeated measures experimental design was used to quantify and compare the mechanical loads, including vertical ground reaction force (vGRF) and the rate of loading (RoL) of the landing phase, of all impact exercises applied in the OPTIMA-Ex trial and to determine the load intensity for each training stage of the impact intervention. Fifteen healthy young adult women aged 18-30 years (mean 23.1 \pm 3.5 years) were recruited (5 trained and 10 untrained). Overall, vGRF was classified as high impact ( $>4$ times body mass [BM]) for all 7 training stages (4.70 \pm 1.89 to $6.79 \pm 2.17$ BM), whereas RoL ranged from $207.01 \pm 175.09$ to $371.52 \pm 393.43 \mathrm{BM} \cdot \mathrm{s}^{-1}$ across the stages. Furthermore, a significant time effect was observed between training stages for vGRF/BM $(p=0.001)$ and RoL $(p<0.001)$. Trained subjects exhibited greater impact loads than untrained subjects for activities at every training stage $(p<0.01)$. We found that impact activities at every stage of the OPTIMA-Ex trial not only met the GRF criteria for high intensity but also exhibited progressive increases in load for successive stages. Furthermore, trained subjects were capable of producing greater impact loads than untrained subjects.
\end{abstract}

Key Words: bone, ground reaction force, mechanical loading, osteogenic exercise

\section{Introduction}

Osteoporosis is a progressive metabolic bone disease characterized by the loss of bone mass and compromised bone strength predisposing to an increased bone fragility and susceptibility to fracture (14). The most common sites of osteoporotic fracture are the spine, hip, and wrist (8). Inadequate accumulation of peak bone mass before skeletal maturity is considered an important risk factor for osteoporosis in old age (23). Physical activity is recognized as one of the most effective lifestyle strategies to maximize peak bone mass during growth (10). As peak bone mass occurs by the end of the third decade (1), young adulthood represents a final opportunity to optimize bone accrual to reduce the likelihood of osteoporosis later in life.

The bone response to exercise is related to the magnitude and rate of strain imposed on it (6). High-impact weight-bearing exercise has been shown to improve bone mass in young and middle-aged adults $(17,36)$. The effect is primarily observed in the lower extremity $(3,15,27)$; however, improvements at the lumbar spine have also been reported (11). It has been suggested that variation in exercise modalities and the corresponding magnitude

Address correspondence to Dr. Benjamin K.Weeks, b.weeks@griffith.edu.au.

Supplemental digital content is available for this article. Direct URL citations appear in the printed text and are provided in the HTML and PDF versions of this article on the journal's Web site (http://journals./ww.com/nsca-jscr).

Journal of Strength and Conditioning Research 00(00)/1-7

(c) 2019 National Strength and Conditioning Association of mechanical loading is a likely reason for the range of outcomes observed after high-impact weight-bearing exercises $(3,7,24)$.

Impact load intensities have previously been classified according to 3 levels (34). "High-intensity" impact loads are considered to apply forces greater than 4 times body mass (BM); "moderate-intensity" loads involve forces between 2 and 4 times BM; whereas "low-intensity" loads are less than 2 times BM (34). Most impact exercise intervention studies fail to report the intensity of loads applied; however, some have estimated impact loads from measures of ground reaction forces (GRF) with large variations of both exercises prescribed and GRF, with reported ranges from 2 to 6 times $\mathrm{BM}(2,3,11)$. Such variation in impact load intensity may explain the inconsistency in bone responses across studies. Impact activities involving accelerations greater than $3.9 \mathrm{~g}$ are positively associated with bone mineral density change at the proximal femur, but accelerations greater than $5.4 \mathrm{~g}$ may be required to effect change at the lumbar spine (30). Such associations between load intensity and the site-specific bone response highlight the importance of incorporating sufficiently high-intensity loads into osteogenic exercise programs and the accurate quantification of such loads in research reports.

The OPTIMA-Ex trial (16) is a 3-arm randomized controlled trial that aims to determine the predominant source of the adaptive bone stimulus-muscle forces or impact loads-by measuring the bone response to the relevant mechanical stimuli in young adult women with low bone mass. An impact-loading training program was included as one of the 3 intervention arms of the trial. The impact-training intervention was designed to be 
a progressive high-intensity (greater than 4 times BM (34)) impact-derived mechanical loading program based on previously published data from our laboratory (33). Over a 10-month training period, subjects in the impact group engaged in a large variety of jumping activities intended to apply landing impacts with varying complexity. In total, 37 different impact-loading exercises were used across 7 successive training stages. All impact exercises fit into 3 defined categories: drop jumps (DJs), unidirectional jumps, and multidirectional jumps, with a further subdivision into bilateral and unilateral landings (prescription of the OPTIMA-Ex trial impact-training program provided in Supplementary Table 1, http://links.lww.com/JSCR/A137). Thus, the impact-loading regime was intended to apply high-intensity, novel, mechanical loads through landing impacts that progressively increase in intensity with each training stage over the intervention period.

The primary aim of the current project was to quantify the intensity of landing impacts for exercises used at each stage of the OPTIMA-Ex trial and establish whether the program effectively applied progressive high-intensity loading. The secondary aim was to examine differences in mechanical loads between trained (OPTIMA-Ex subjects) and untrained (novice) subjects.

\section{Methods}

\section{Experimental Approach to the Problem}

A randomized repeated measures experimental design was used to quantify and compare the vertical ground reaction force (vGRF) relative to $\mathrm{BM}$ and the rate of loading $\left(\mathrm{RoL}, \mathrm{BM} \cdot \mathrm{s}^{-1}\right.$ ) for the landing phase of each impact exercise at each successive training stage used in the OPTIMA-Ex impact-training program. Both trained and untrained subjects were tested to determine the influence of training status on the intensity of the mechanical loads achieved during each activity. The current study characterizes and reports a subset of data collected in the course of the OPTIMA-Ex trial. Primary outcomes of the randomized controlled OPTIMA-Ex trial are to be published in a subsequent manuscript.

\section{Subjects}

Fifteen healthy young adult women aged 18-30 years were recruited for the study (age $=23.2 \pm 3.5$ years; height $=167 \pm 6$ $\mathrm{cm}$; body mass $=59.1 \pm 8.1 \mathrm{~kg}$; and body mass index $[\mathrm{BMI}]=$ $\left.21.0 \pm 2.8 \mathrm{~kg} \cdot \mathrm{m}^{-2}\right)$. Five women had completed the 10 -month OPTIMA-Ex impact-training intervention (age $=21.7 \pm 3.8$ years; height $=166 \pm 7 \mathrm{~cm}$; body mass $=51.2 \pm 4.9 \mathrm{~kg}$; and $\mathrm{BMI}$ $=18.5 \pm 1.0 \mathrm{~kg} \cdot \mathrm{m}^{-2}$ ), whereas 10 women were untrained in impact exercises $($ age $=23.8 \pm 3.2$ years; height $=168 \pm 7 \mathrm{~cm}$; body mass $=63.2 \pm 6.2 \mathrm{~kg}$; and BMI $=22.3 \pm 2.5 \mathrm{~kg} \cdot \mathrm{m}^{-2}$ ), having had no previous impact training. Data were pooled for all subjects to examine the landing intensities of all impact exercises and differences between the activities used in successive training stages of the OPTIMA-Ex trial. However, between-group differences were also examined for each successive training stage. Subjects were excluded if they had a recent lower-limb injury (within the past 6 months) or surgery (within the past 12 months). Each subject was verbally informed of the purpose, procedure, benefits, and risks of the investigation, and that they could withdraw at any time without prejudice, before providing written informed consent. All experimental procedures were approved by the Griffith University Human Research Ethics Committee (Approval number: PES/20/11/HREC and GU Ref: 2015/775).

\section{Procedures}

Each subject was tested on 3 separate nonconsecutive days to capture the 3 training domains of drop jumps, unidirectional impacts, and multidirectional impacts. The sectioned block testing approach with rest days was adopted to minimize the influence of fatigue because of the large number of exercises examined in the study. Subjects were asked to abstain from caffeine and exercise on the day of testing, and each testing session was scheduled at the same time of each test day. A randomized sequence of impact exercises compiled using an online random number generator to control for exercise order was used to minimize the influence of fatigue. Before testing, height and body mass were measured using a wall-mounted stadiometer $(S+M$, Aaxis Pacific, Blacktown, Australia) and digital scale (TANITA Co., Tokyo, Japan).

Before testing, subjects performed a standardized 3-minute warm-up at $50 \mathrm{~W}$ on a cycle ergometer (Monark 818; Vansbro, Sweden) at their preferred cadence. During the warm-up, a standardized verbal overview of the exercises was provided along with instructions to land as stiff-legged as possible on their heels in a firm but comfortable manner. Each impact exercise was demonstrated by the investigator before the subjects completed practice attempts to ensure safe and correct landing technique was achieved. A series of drop jumps, unidirectional and multidirectional impacts were then performed in accordance with the impact exercises as part of the OPTIMA-Ex trial (Table 1). Each subject completed a set of 3 attempts of each impact exercise (or each component of the exercises in the case of unilateral and multidirectional impacts). All impact exercises were performed barefoot as per the OPTIMA-Ex trial to avoid the confounding influence of different footwear between subjects. Landing impacts were recorded in a single motion capture, and all force plates were zeroed before each set to minimize errors in data collection. All subjects received a 1-minute rest between exercise sets to mitigate the influence of muscle fatigue.

Data Analysis. All landing impacts were collected using a single $86.5 \times 56.5-\mathrm{cm}$ Advanced Medical Technologies Inc. (AMTI) OR6 series force plate (AMTI Inc., Watertown, MA, USA), sampling at $1,000 \mathrm{~Hz}$ installed in the floor. Force plate data were recorded using Vicon Nexus 2.5 software (Vicon, Oxford Metrics, Oxford, United Kingdom). The raw recorded data were exported at the end of each testing session and processed in a custom-written software program using MATLAB version R2017a (MathWorks, Inc., Natick, MA, USA). Vertical GRF was defined as the peak force magnitude of the passive impact load endured during the landing phase of each activity, and RoL was defined as the force applied over the time from initial contact to the peak force magnitude of the passive impact peak during the landing phase. All GRFs were normalized to $\mathrm{BM}$, and the average peak landing vGRFs (BM) and RoL (BM-s $\left.{ }^{-1}\right)$ were calculated for each exercise from 3 successful trials. Impact exercises were then stratified into their respective training stages as per the OPTIMA-Ex trial protocol (Table 1). The osteogenic index (OI) of a single session from each training stage was calculated using the method described by Turner and Robling (OI [1 session] = peak ground reaction force $(\mathrm{BM}) \times \ln$ [number of loading cycles +1 ]) $(29)$.

\section{Statistical Analyses}

Descriptive statistics (mean $\pm S D$ ) were computed for anthropometrics, peak impact phase vGRF/BM and RoL. Shapiro-Wilks 
OPTIMA-Ex trial impact exercises, training stages, and associated acronyms.

\begin{tabular}{|c|c|c|c|c|c|}
\hline \multirow{2}{*}{$\begin{array}{l}\text { Training } \\
\text { stage }\end{array}$} & \multirow{2}{*}{$\begin{array}{l}\text { Drop jump } \\
\text { Bilateral }\end{array}$} & \multicolumn{2}{|c|}{ Unidirectional } & \multicolumn{2}{|c|}{ Multidirectional } \\
\hline & & Bilateral & Unilateral & Bilateral & Unilateral \\
\hline Stage 1 & $15 \mathrm{~cm}$ (DJ 15) & Squat jump (SJ) & Bounding (bound) & Lateral cone jump (LCJ) & Lateral ski jump (LSkJ) \\
\hline Stage 2 & $15 \mathrm{~cm}$ (DJ 15) & Broad jump (BJ) & Hop for distance (hop) & $\begin{array}{l}\text { Lateral box jump on } 15-\mathrm{cm} \\
\text { box (LBJ) }\end{array}$ & Lateral cone hop (LCH) \\
\hline Stage 3 & $30 \mathrm{~cm}$ (DJ 30) & $\begin{array}{c}\text { Hurdle jump } \\
\text { consecutive (HJ) }\end{array}$ & $\begin{array}{l}\text { 30-cm box march } \\
\text { alternate legs (BM) }\end{array}$ & $\begin{array}{l}\text { Lateral hurdle jump } \\
\text { consecutive (LHJ) }\end{array}$ & $\begin{array}{l}\text { Lateral box shuffle on } \\
15-\mathrm{cm} \text { box (LBSh) }\end{array}$ \\
\hline Stage 4 & $40 \mathrm{~cm}$ (DJ 40) & $\begin{array}{l}\text { Hurdle jump stick } \\
\text { landings (HJSL) }\end{array}$ & 15-cm box hop (BH) & Zig-zag cone jumps $\left(45^{\circ} \mathrm{CJ}\right)$ & $\begin{array}{l}\text { Lateral ladder hop } \\
\text { consecutive (LLH) }\end{array}$ \\
\hline Stage 5 & $50 \mathrm{~cm}$ (DJ 50) & $\begin{array}{l}\text { 30-cm depth jump into } \\
\text { broad jump (DJSL) }\end{array}$ & $\begin{array}{c}\text { Hurdle hop } \\
\text { consecutive (HH) }\end{array}$ & Lateral box broad jump (LBBJ) & $\begin{array}{l}\text { Zig-zag cone hop for distance } \\
\text { consecutive }\left(45^{\circ} \mathrm{CH}\right)\end{array}$ \\
\hline Stage 6 & $60 \mathrm{~cm}$ (DJ 60) & $\begin{array}{l}\text { 30-cm depth jump for } \\
\text { height (DJH) }\end{array}$ & $\begin{array}{l}\text { Hurdle hop stick } \\
\text { landings (HHSL) }\end{array}$ & $\begin{array}{l}\text { 15-cm multidirectional box } \\
\text { jump (on and off) (MDBJ) }\end{array}$ & $\begin{array}{l}\text { Zig-zag cone hop for height stick } \\
\left.\text { landing ( } 45^{\circ} \mathrm{CHSL}\right)\end{array}$ \\
\hline Stage 7 & 80 cm (DJ 80) & $\begin{array}{l}\text { 30-cm depth jump } \\
\text { onto 15-cm platform (DJP) }\end{array}$ & $\begin{array}{l}\text { 30-cm depth hops for } \\
\text { height (DH) }\end{array}$ & $\begin{array}{l}\text { 30-cm multidirectional depth } \\
\text { jump (DJMD) }\end{array}$ & Multidirectional ladder hop (MDLH) \\
\hline
\end{tabular}

tests were used to determine normality of data. For whole-group analyses, 1-way repeated measures analysis of variance (ANOVA) and Friedman's test were performed for each impact exercise type and training stage. Between-group differences on the basis of exercise expertise were also examined with 1-way repeated measures ANOVA and Kruskal-Wallis tests for normally and non-normally distributed data, respectively. Subsequently, each impact-loading exercise was analyzed separately in their respective domains of drop jump, unidirectional (bilateral and unilateral) and multidirectional (bilateral and unilateral) for both vGRF and RoL. Significant main effects were further analyzed with Bonferroni-adjusted pairwise comparisons for normally distributed data; however, for non-normally distributed data, Dunn's test was used. Statistical significance was assumed at $p<$ 0.05 with partial eta-squared representing effect size, along with observed power for normally distributed data, whereas chisquare results are presented for non-normally distributed data. All analyses were performed using SPSS v.22 (IBM Inc., Chicago, IL, USA). Sample size was based on previous reports of vGRF data for drop jump landings. Using a 2-tailed test with $80 \%$ power and $\alpha=0.05$, a total of 10 subjects were required to detect differences $0.47 \pm 0.22 \mathrm{BM}$ between jump levels (DJ 20 and DJ $40 \mathrm{~cm} \mathrm{(20)),} \mathrm{whereas} 5$ subjects were required in each group to detect differences of $13.29 \pm 6.28 \mathrm{~N} \cdot \mathrm{kg}^{-1}$ between trained and untrained subjects (DJ $60 \mathrm{~cm} \mathrm{(25)).}$

\section{Results}

Age and height were similar between groups; however, women in the untrained group were heavier $(p=0.002)$ and thus had a greater BMI $(p=0.007)$. Despite the between-group differences, both groups were within the normal healthy BMI range (35).

For the whole group, vGRF results were normally distributed; however, RoL results were not. For vGRF, differences were observed for vGRF across training stages $(p<0.001$; effect size $=$ 0.682 ; power $=1.000)$ and for OI $(p<0.001$; effect size $=0.677$; power $=1.000)($ Table 2$)$. Similarly, differences were observed for RoL across training stages $\left(\chi^{2}(5)=52.33, p=0.000\right)$. Follow-up pairwise comparisons indicated that differences occurred between many training stages of the OPTIMA-Ex trial for $\mathrm{vGRF} / \mathrm{BM}(p=0.001-0.028)$ and $\operatorname{RoL}(p=0.001-0.049)$. In most cases, each stage $\mathrm{vGRF} / \mathrm{BM}$ and $\mathrm{RoL}$ were significantly greater than the preceding ones (Figure 1A, B). Furthermore,
$\operatorname{vGRF}(p=0.001-0.007)$ (Figure 1C) and $\operatorname{RoL}(p=0.001-0.002)$ (Figure 1D) were greater in trained subjects than in untrained subjects at every training stage.

Each impact-loading exercise when separated into their respective exercise domains was analyzed for both the magnitude and rate as well as examined for between-group differences (Tables 3 and 4). Differences were observed for vGRF measures of drop jump landings $(p=0.001$; effect size $=0.894$; power $=$ $1.000)$, unidirectional bilateral impacts $(p=0.001$; effect size $=$ 0.952 ; power $=1.000)$ and unilateral impacts $(p=0.008$; effect size $=0.804$; power $=0.927)$, and multidirectional unilateral impacts $(p=0.001$; effect size $=0.914$; power $=1.000)$, but not bilateral impacts $(p=0.139$, effect size $=0.594$; power $=0.479)$. Furthermore, differences were observed for RoL for unidirectional bilateral $\left(\chi^{2}(5) 48.286, p<0.001\right)$ and unilateral $\left(\chi^{2}(5)\right.$ $47.786, p<0.001)$, multidirectional bilateral $\left(\chi^{2}(5) 37.600, p=\right.$ $>0.001)$ and unilateral $\left(\chi^{2}(5) 51.22, p<0.001\right)$, and drop jump loadings $\left(\chi^{2}(5) 25.362, p<0.001\right)$.

\section{Discussion}

The aim of the current work was to quantify the landing impact intensities for exercises used in the OPTIMA-Ex trial and to examine load progression across incremental training stages. In addition, we sought to determine whether training influenced the mechanical loads experienced. Overall, vGRF was greater than 4 times BM for all 7 training stages ranging from 4.70 to 6.79 times $\mathrm{BM}$ and increasing over time, suggesting that the impact intervention arm of the OPTIMA-Ex trial is both high intensity and progressive in nature. The largest vGRFs were recorded for drop jump landings with magnitudes reaching 9.48 times BM for the $80-\mathrm{cm}$ box height, whereas the fastest RoL was recorded for the broad jump landing at $654.56 \mathrm{BM}$ per second. Furthermore, differences were observed between trained and untrained subjects at each training stage for vGRF and RoL, with trained subjects capable of producing larger and faster loads for all activities.

Findings from animal research have established that the most osteogenic loads are those that induce high-magnitude bone strains (22) at high loading rates $(21,28)$. When designing a bone-targeted exercise program, not only does the magnitude and rate need to be maximized but so too does the novelty and progression of loading, as bone accommodates to customary mechanical stimuli (29). Indeed, the recent position statement by 


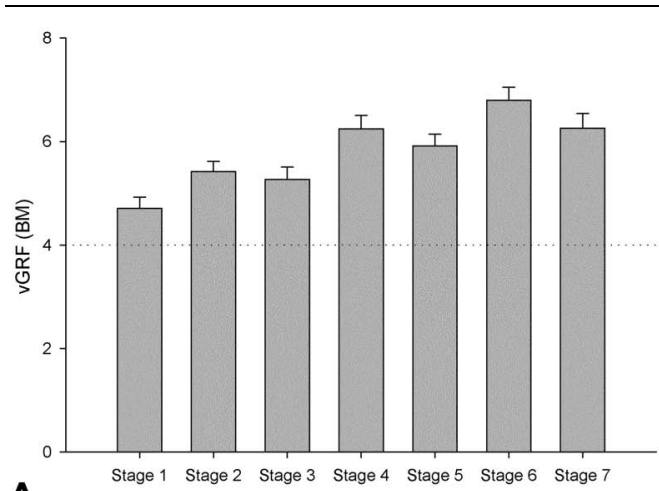

A

Training Stage

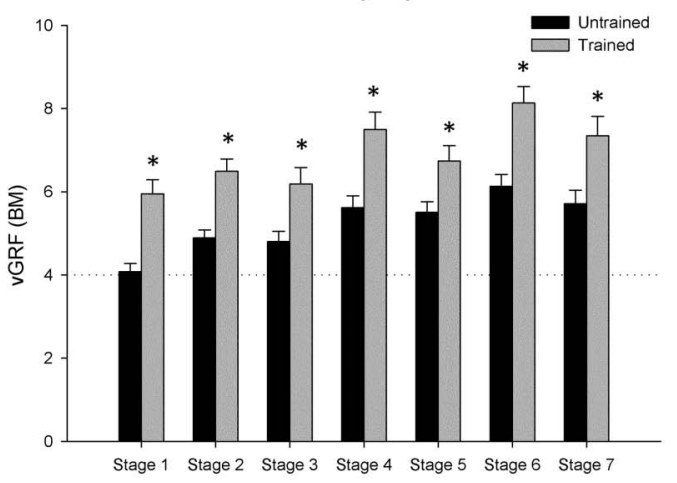

Training Stage

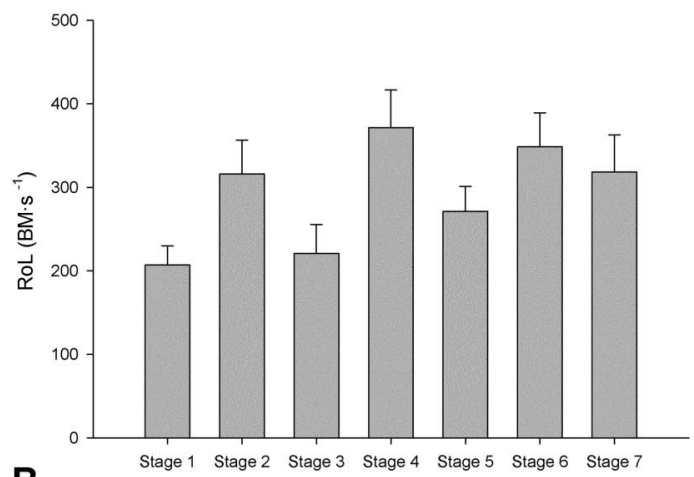

B

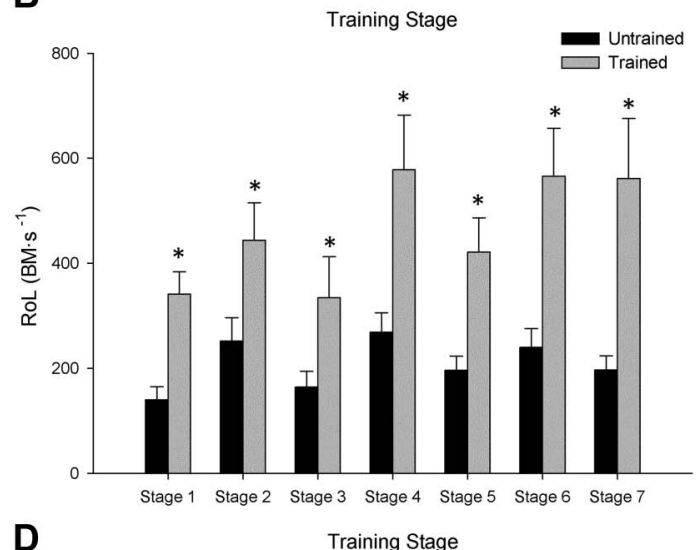

Figure 1. Average $( \pm S D$ ) vertical ground reaction force (vGRF) and rate of loading (RoL) for the landing phase of impact activities at each training stage with reference to the high-intensity loading threshold (dotted line) $(n=15)$. A) Whole-group vGRF; (B) whole-group RoL; (C) between-group differences vGRF * $p<0.01$; and (D) between-group differences RoL ${ }^{\star} p<$ 0.02. $\mathrm{BM}=$ body mass.

Exercise and Sports Science Australia recommends progressively increasing impact activities that include jumps, hops and multidirectional weight-bearing exercises for prevention and management of osteoporosis (4). Our findings about the impact intervention of the OPTIMA-Ex trial support the fundamental principles and current recommendations for osteogenic exercise prescription.

Landing from a drop jump has been well characterized from a mechanical loading perspective. Somewhat intuitively, most reports have demonstrated an increase in peak GRF with increasing drop height $(5,18-20)$. Similarly, our findings demonstrate that vGRF increases with drop height; however, we observed much higher magnitudes than those previously reported

\section{Table 2}

Osteogenic index (OI) for a single session in each training stage (mean $\pm S D$ ).

\begin{tabular}{lccc}
\hline & \multicolumn{3}{c}{ Osteogenic index (per session) } \\
\cline { 2 - 4 } Training stage & Untrained $(\boldsymbol{n = 1 0})$ & Trained $(\boldsymbol{n}=\mathbf{5})$ & Total $(\boldsymbol{n = 1 5})$ \\
\hline Stage 1 & $19.58 \pm 2.50$ & $28.55 \pm 4.41 \dagger$ & $22.56 \pm 5.36$ \\
Stage 2 & $23.43 \pm 3.25$ & $31.13 \pm 5.98 \dagger$ & $26.00 \pm 5.58^{\star}$ \\
Stage 3 & $23.04 \pm 4.98$ & $29.69 \pm 6.93 \dagger$ & $25.26 \pm 6.34$ \\
Stage 4 & $26.94 \pm 5.19$ & $35.96 \pm 3.22 \dagger$ & $29.95 \pm 6.29^{\star}$ \\
Stage 5 & $26.39 \pm 4.86$ & $32.33 \pm 5.15 \dagger$ & $28.37 \pm 5.58$ \\
Stage 6 & $29.39 \pm 6.54$ & $39.01 \pm 4.77 \dagger$ & $32.60 \pm 7.48^{\star}$ \\
Stage 7 & $27.38 \pm 6.03$ & $35.24 \pm 4.98 \dagger$ & $30.0 \pm 6.72^{\star}$ \\
\hline
\end{tabular}

${ }^{*} p<0.05$ indicates difference to previous training stage.

$\dagger p<0.05$ indicates between-group differences for trained and untrained subjects.
(20,31,32). For instance, previous reports showed magnitudes of 3.3-5.4 BM for drop landings from heights of 30-90 cm (31). However, this difference in magnitude may be due to current study instructions to land in a comfortable but "stiff-legged" position emphasizing a heel impact where feasible. From an osteogenic exercise prescription perspective, progressive height drop jump landings seem to impart the highest magnitude of load and offer a convenient method of load intensity adjustment as the intervention progresses. Naturally, discomfort and the risk of injury will ultimately limit the loading potential from this strategy.

Effective osteogenic loading is not only dependent on the magnitude of load but also dependent on the rate at which the load is applied (29). Previous research in healthy, young adults aged $18-30$ has demonstrated that walking (1.2 $\mathrm{BM})$ produces loading rates of $8.4 \mathrm{BM} \cdot \mathrm{s}^{-1}$, running $(2.6 \mathrm{BM})$ produces a rate of $46.9 \mathrm{BM} \cdot \mathrm{s}^{-1}$, whereas a foot stomp (4.6 BM) loads at a rate of $473.6 \mathrm{BM} \cdot \mathrm{s}^{-1}$ (33). The highest rate in this study was recorded for broad jump landings $(6.62 \mathrm{BM})$ at 654.56 $\mathrm{BM} \cdot \mathrm{s}^{-1}$, whereas the lowest loading rate was recorded for lateral ski jump landings $(2.88 \mathrm{BM})$ at $80.02 \mathrm{BM} \cdot \mathrm{s}^{-1}$. In fact, our RoL results for lateral ski jump landings mirror that of previously reported single leg side step landings (2.9 BM) producing $117.4 \mathrm{BM} \cdot \mathrm{s}^{-1}(33)$. For our highest magnitude activity of $80-\mathrm{cm}$ drop jump $(9.48 \mathrm{BM})$, a rate of $277.17 \mathrm{BM} \cdot \mathrm{s}^{-1}$ was observed. As landings that produce the highest magnitudes do not necessarily elicit the highest loading rate, both factors need to be taken into consideration when designing an osteogenic exercise program. 
Table 3

Vertical ground reaction force (vGRF) expressed as body mass

(BM) for the landing phase of each impact exercise (mean $\pm S D)$.*

Group

\begin{tabular}{|c|c|c|c|}
\hline Impact exercise & Untrained $(n=10)$ & Trained $(n=5)$ & Total $(n=15)$ \\
\hline \multicolumn{4}{|l|}{ Drop bilateral } \\
\hline DJ 15 & $4.80 \pm 0.91$ & $7.10 \pm 2.19 \dagger$ & $5.57 \pm 1.78$ \\
\hline DJ 30 & $6.55 \pm 1.91$ & $7.97 \pm 1.89$ & $7.02 \pm 1.96$ \\
\hline DJ 40 & $7.02 \pm 1.56$ & $8.94 \pm 1.62 \dagger$ & $7.67 \pm 1.79$ \\
\hline DJ 50 & $7.62 \pm 1.67$ & $9.09 \pm 1.39$ & $8.11 \pm 1.70$ \\
\hline DJ 60 & $8.32 \pm 1.46$ & $9.63 \pm 1.44$ & $8.75 \pm 1.53$ \\
\hline DJ 80 & $8.98 \pm 0.96$ & $10.47 \pm 0.76 \dagger$ & $9.48 \pm 1.14$ \\
\hline \multicolumn{4}{|c|}{ Unidirectional bilateral } \\
\hline SJ & $5.40 \pm 1.83$ & $7.72 \pm 0.94 \dagger$ & $6.18 \pm 1.91$ \\
\hline BJ & $6.32 \pm 2.08$ & $7.23 \pm 1.63$ & $6.62 \pm 1.93$ \\
\hline HJ & $5.08 \pm 0.91$ & $7.37 \pm 3.07 \dagger$ & $5.85 \pm 2.11$ \\
\hline HJSL & $7.55 \pm 1.68$ & $9.80 \pm 1.87 \dagger$ & $8.31 \pm 2.00$ \\
\hline DJSL & $5.78 \pm 1.87$ & $7.13 \pm 1.76$ & $6.23 \pm 1.89$ \\
\hline DJH & $6.59 \pm 1.55$ & $9.13 \pm 1.53 \dagger$ & $7.45 \pm 1.94$ \\
\hline DJP & $5.35 \pm 2.18$ & $7.94 \pm 1.99 \dagger$ & $6.22 \pm 2.41$ \\
\hline \multicolumn{4}{|c|}{ Unidirectional unilateral } \\
\hline Bound & $3.74 \pm 0.64$ & $3.78 \pm 0.75$ & $3.72 \pm 0.62$ \\
\hline Hop & $4.47 \pm 0.96$ & $5.77 \pm 0.80 \dagger$ & $4.91 \pm 1.08$ \\
\hline BM & $4.06 \pm 1.07$ & $4.84 \pm 0.511$ & $4.32 \pm 0.97$ \\
\hline $\mathrm{BH}$ & $3.79 \pm 1.48$ & $6.26 \pm 2.02 \dagger$ & $4.61 \pm 2.00$ \\
\hline $\mathrm{HH}$ & $4.67 \pm 1.54$ & $6.33 \pm 1.24$ & $5.23 \pm 1.62$ \\
\hline HHSL & $2.59 \pm 1.87$ & $7.73 \pm 1.26 \dagger$ & $6.30 \pm 1.94$ \\
\hline $\mathrm{DH}$ & $4.82 \pm 1.60$ & $7.03 \pm 0.92 \dagger$ & $5.55 \pm 1.74$ \\
\hline \multicolumn{4}{|c|}{ Multidirectional bilateral } \\
\hline LCJ & $3.77 \pm 0.51$ & $7.44 \pm 1.30 \dagger$ & $4.99 \pm 1.96$ \\
\hline LBJ & $4.99 \pm 0.64$ & $7.16 \pm 1.96 \dagger$ & $5.71 \pm 1.57$ \\
\hline LHJ & $4.65 \pm 1.01$ & $6.41 \pm 1.51 \dagger$ & $5.24 \pm 1.43$ \\
\hline $45^{\circ} \mathrm{CJ}$ & $4.85 \pm 0.92$ & $7.31 \pm 1.33 \dagger$ & $5.67 \pm 1.43$ \\
\hline LBBJ & $4.89 \pm 0.85$ & $6.31 \pm 1.25 \dagger$ & $5.37 \pm 1.18$ \\
\hline MDBJ & $4.87 \pm 1.71$ & $8.18 \pm 1.31 \dagger$ & $5.98 \pm 2.23$ \\
\hline DJMD & $5.36 \pm 1.68$ & $6.37 \pm 2.48$ & $5.70 \pm 1.95$ \\
\hline \multicolumn{4}{|c|}{ Multidirectional unilateral } \\
\hline LSkJ & $2.63 \pm 0.45$ & $3.38 \pm 0.56 \dagger$ & $2.88 \pm 0.60$ \\
\hline $\mathrm{LCH}$ & $3.55 \pm 0.43$ & $4.94 \pm 0.97 \dagger$ & $4.02 \pm 0.92$ \\
\hline LBSh & $3.66 \pm 0.97$ & $3.99 \pm 1.04$ & $3.77 \pm 0.96$ \\
\hline LLH & $4.48 \pm 1.03$ & $4.92 \pm 0.94$ & $4.62 \pm 0.99$ \\
\hline $45^{\circ} \mathrm{CH}$ & $4.27 \pm 0.80$ & $4.81 \pm 0.91$ & $4.45 \pm 0.85$ \\
\hline $45^{\circ} \mathrm{CHSL}$ & $5.34 \pm 1.57$ & $6.14 \pm 1.70$ & $5.61 \pm 1.60$ \\
\hline MDLH & $4.11 \pm 1.47$ & $4.98 \pm 1.41$ & $4.40 \pm 1.47$ \\
\hline
\end{tabular}

${ }^{*}$ DJ 15 = drop jump off 15-cm box; DJ $30=$ drop jump off 30-cm box; DJ $40=$ drop jump off 40-cm box; DJ 50 = drop jump off 50-cm box; DJ 60 = drop jump off 60-cm box; DJ 80 drop jump off 80-cm box; $\mathrm{SJ}=$ squat jump; $\mathrm{BJ}=$ broad jump; $\mathrm{HJ}=$ hurdle jump (consecutive); HJSL = hurdle jump stick landings; DJSL = depth jump from 30-cm box into broad jump; DJH = depth jump for height off 30-cm box; DJP = depth jump off 30-cm box onto 15-cm platform; Bound = bounding; Hop = hop for distance; $\mathrm{BM}=$ box march on 30-cm box; $\mathrm{BH}=$ box hop onto 15-cm box; $\mathrm{HH}=$ hurdle hop (consecutive); HHSL = hurdle hop sticking the landing; $\mathrm{DH}$ = depth hop for height; LCJ $=$ lateral cone jump; $\mathrm{LBJ}=$ lateral box jump on $15-\mathrm{cm}$ box; $\mathrm{LHJ}=$ lateral hurdle jump (consecutive), $45^{\circ} \mathrm{CJ}=$ zig-zag cone jump; LBBJ = lateral box broad jump; MDBJ = multidirectional box jump on and off 15-cm box; DJMD = depth jump of $30 \mathrm{~cm}$ into lateral jump; LSkJ = lateral ski jump; LCH = lateral cone hop; LBSh = lateral box shuffle; $\mathrm{LLH}=$ lateral ladder hop (consecutive); $45^{\circ} \mathrm{CH}=$ zigzag cone hop for distance (consecutive); $45^{\circ} \mathrm{CHSL}=$ zig-zag cone hop for height stick landing; MDLH $=$ multidirectional ladder hop.

$\dagger p<0.05$ indicates between-group differences for trained and untrained subjects.

We observed greater peak landing impacts for the trained group compared with the untrained group at every training stage of the OPTIMA-Ex protocol. Competitive gymnasts produce $33 \%$ higher peak magnitudes at a $60-\mathrm{cm}$ drop height and $34 \%$ higher peak magnitudes at a $90-\mathrm{cm}$ drop height than recreational athletes (25). The results of the OPTIMA-Ex trained group reflect those of gymnasts during drop jumps from different heights with reported ranges of 3.9-11.0 BM for drop heights ranging from 32 to $128 \mathrm{~cm} \mathrm{(18).} \mathrm{These} \mathrm{similarities} \mathrm{in} \mathrm{results} \mathrm{may} \mathrm{be} \mathrm{a} \mathrm{result} \mathrm{of} \mathrm{the}$ impact-trained group's confidence and familiarization to the instructed landings strategy. A study of male athletes landing from a drop height of $30.48 \mathrm{~cm}$ found that "stiff-legged" landing on heels indeed produced significantly higher GRFs than either of the 3 other landing instructions (i.e., natural landing knees bent, natural landing stiff leg, and forefoot landing stiff leg) (26). Thus, the difference in the impact magnitudes observed between trained and untrained groups may reflect their ability to achieve the necessary "stiff-legged" landing.

Although the primary aim of the current work was to quantify the landing impacts for exercises applied in the OPTIMA-Ex trial, perhaps the most novel aspect of our study was the extensive range of different impact exercises examined. Thirty-seven different exercises were examined for magnitude and RoL across 3 landing domains of drop jump landings, unidirectional and multidirectional impacts with subdivisions of bilateral and unilateral landings. Previous reports have typically quantified considerably fewer exercises (i.e., less than 10) $(9,31)$ or looked specifically at 1 training domain (i.e., drop jump landings) $(18,19,25)$. Thus, our work represents the most comprehensive characterization of impact exercise load magnitudes and rates. Such a resource is valuable to clinicians and researchers developing bone-targeted exercise programs.

Several limitations warrant acknowledgement. First, we used vGRF as a surrogate estimate of skeletal load, and therefore, the actual stresses at the joint level and microstrain experienced at the bone-tissue level can only be inferred. The direct measurement of strain from the skeleton during exercise is highly problematic, not only due to logistical challenges related to the invasive nature of the procedure but also due to the complexity of strain distribution on and within whole bones. As strong relationships between vGRF and a range of landing activities have previously been demonstrated $(5,13,18)$, we believe that the measurement of vGRFs during exercise provides an acceptable index of musculoskeletal loading. Second, as only vertical GRFs were measured, our results do not account for loads experienced in other directions. We limited our investigation to vGRF only because of the typical landing nature and predominantly axial loads of exercises included in the OPTIMA-Ex impact program. Third, although our overall sample seems "moderate" in size and even "low" for between-group comparisons, our study numbers were derived from previous reports $(12,13,18,20,25,31)$. Post hoc power analyses further support the number of subjects examined. In the results, a significant difference was observed in BM and BMI between trained and untrained subjects; however, both groups were considered within the normal BMI range $\left(18.5-24.9 \mathrm{~kg} \cdot \mathrm{m}^{-2}\right)$ (35). Furthermore, all landings were normalized to BM to mitigate its influence on the results. Finally, only kinetic data was collected, and therefore, we can only hypothesize the effect that varying movement patterns and landing strategies may have had on our results.

In conclusion, our findings suggest that the impact intervention of the OPTIMA-Ex trial can be considered a progressive and high-intensity impact-loading exercise program. Furthermore, our work demonstrates that as exercise subjects become more experienced and familiar with the performance of impact exercises, progressively increasing load magnitudes are feasible. Our findings provide a valuable reference for load magnitudes and rates for a wide variety of landing exercises to assist clinicians and researchers in their efforts to design osteogenic exercise programs. 
Table 4

Rate of loading (RoL) displayed as body mass per second $\left(B M \cdot s^{-1}\right)$ for the landing phase of each impact exercise (mean $\left.\pm S D\right) .^{*}$

\begin{tabular}{|c|c|c|c|}
\hline \multirow[b]{2}{*}{ Impact exercise } & \multicolumn{3}{|c|}{ Group } \\
\hline & Untrained $(n=10)$ & Trained $(n=5)$ & Total $(n=15)$ \\
\hline \multicolumn{4}{|l|}{ Drop bilateral } \\
\hline DJ 15 & $131.73 \pm 162.86$ & $287.61 \pm 236.51 \dagger$ & $183.69 \pm 197.02$ \\
\hline DJ 30 & $228.74 \pm 284.32$ & $228.71 \pm 122.31$ & $228.73 \pm 237.15$ \\
\hline DJ 40 & $265.41 \pm 299.79$ & $232.47 \pm 94.67$ & $254.43 \pm 246.16$ \\
\hline DJ 50 & $266.99 \pm 278.97$ & $234.82 \pm 76.76$ & $256.26 \pm 227.95$ \\
\hline DJ 60 & $316.26 \pm 303.47$ & $284.28 \pm 78.10$ & $305.60 \pm 247.37$ \\
\hline DJ 80 & $253.39 \pm 94.58$ & $324.73 \pm 42.13$ & $277.17 \pm 86.42$ \\
\hline \multicolumn{4}{|c|}{ Unidirectional bilateral } \\
\hline SJ & $94.29 \pm 52.70$ & $302.0 \pm 164.21 \dagger$ & $163.55 \pm 140.60$ \\
\hline BJ & $582.52 \pm 464.73$ & $798.64 \pm 486.17$ & $654.56 \pm 466.36$ \\
\hline HJ & $125.78 \pm 79.27$ & $417.59 \pm 385.46$ & $223.05 \pm 258.39$ \\
\hline HJSL & $268.37 \pm 163.05$ & $943.48 \pm 623.17 \dagger$ & $493.41 \pm 486.37$ \\
\hline DJSL & $587.26 \pm 433.17$ & $547.33 \pm 325.33$ & $573.95 \pm 388.90$ \\
\hline DJH & $208.50 \pm 174.92$ & $722.87 \pm 462.76 \dagger$ & $379.96 \pm 379.28$ \\
\hline DJP & $391.12 \pm 307.38$ & $989.06 \pm 1,006.63$ & $589.44 \pm 659.19$ \\
\hline \multicolumn{4}{|c|}{ Unidirectional unilateral } \\
\hline Bound & $379.35 \pm 226.37$ & $416.87 \pm 180.98$ & $391.87 \pm 206.48$ \\
\hline Hop & $402.52 \pm 238.34$ & $572.16 \pm 337.00$ & $459.07 \pm 275.35$ \\
\hline BM & $71.84 \pm 34.28$ & $164.15 \pm 82.49$ & $102.61 \pm 68.76$ \\
\hline $\mathrm{BH}$ & $391.82 \pm 413.74$ & $865.39 \pm 771.39$ & $549.68 \pm 577.46$ \\
\hline $\mathrm{HH}$ & $106.82 \pm 66.60$ & $432.76 \pm 347.80 \dagger$ & $215.47 \pm 250.41$ \\
\hline HHSL & $186.43 \pm 147.39$ & $689.62 \pm 415.43 \dagger$ & $354.16 \pm 351.51$ \\
\hline $\mathrm{DH}$ & $98.97 \pm 70.56$ & $481.67 \pm 281.16 \dagger$ & $226.53 \pm 246.29$ \\
\hline \multicolumn{4}{|c|}{ Multidirectional bilateral } \\
\hline LCJ & $53.35 \pm 12.70$ & $410.57 \pm 301.94 \dagger$ & $172.42 \pm 237.77$ \\
\hline LBJ & $79.05 \pm 25.39$ & $198.59 \pm 126.21 \dagger$ & $118.89 \pm 91.48$ \\
\hline LHJ & $75.06 \pm 29.03$ & $176.25 \pm 109.71 \dagger$ & $108.79 \pm 80.12$ \\
\hline $45^{\circ} \mathrm{CJ}$ & $270.38 \pm 173.70$ & $538.97 \pm 188.92 \dagger$ & $359.92 \pm 216.27$ \\
\hline LBBJ & $105.46 \pm 64.04$ & $378.53 \pm 246.37 \dagger$ & $196.48 \pm 194.25$ \\
\hline MDBJ & $66.32 \pm 37.61$ & $238.71 \pm 158.97 \dagger$ & $123.79 \pm 123.31$ \\
\hline DJMD & $175.61 \pm 222.77$ & $459.55 \pm 311.93$ & $270.26 \pm 280.89$ \\
\hline \multicolumn{4}{|c|}{ Multidirectional unilateral } \\
\hline LSkJ & $31.63 \pm 11.38$ & $176.81 \pm 195.61 \dagger$ & $80.02 \pm 126.62$ \\
\hline LCH & $55.25 \pm 13.76$ & $413.18 \pm 581.98 \dagger$ & $174.56 \pm 356.92$ \\
\hline LBSh & $62.53 \pm 33.30$ & $183.11 \pm 167.53$ & $102.72 \pm 110.42$ \\
\hline LLH & $145.77 \pm 139.30$ & $308.88 \pm 300.19 \dagger$ & $200.14 \pm 211.08$ \\
\hline $45^{\circ} \mathrm{CH}$ & $302.90 \pm 189.95$ & $558.49 \pm 414.48$ & $388.10 \pm 296.36$ \\
\hline $45^{\circ} \mathrm{CHSL}$ & $421.66 \pm 366.91$ & $808.39 \pm 697.76 \dagger$ & $550.57 \pm 511.14$ \\
\hline MDLH & $117.23 \pm 116.69$ & $403.98 \pm 416.97 \dagger$ & $212.81 \pm 279.29$ \\
\hline
\end{tabular}

${ }^{*}$ DJ 15 = drop jump off 15-cm box; DJ $30=$ drop jump off 30-cm box; DJ $40=$ drop jump off 40-cm box; DJ $50=$ drop jump off 50-cm box; DJ $60=$ drop jump off 60 -cm box; DJ 80 drop jump off 80 -cm box; $\mathrm{SJ}=$ squat jump; $\mathrm{BJ}=$ broad jump; $\mathrm{HJ}=$ hurdle jump (consecutive); HJSL = hurdle jump stick landings; DJSL = depth jump from 30-cm box into broad jump; DJH = depth jump for height off 30-cm box; DJP = depth jump off 30-cm box onto 15-cm platform; Bound = bounding; Hop = hop for distance; BM = box march on 30-cm box; BH = box hop onto 15-cm box; HH = hurdle hop (consecutive); $\mathrm{HHSL}=$ hurdle hop sticking the landing; $\mathrm{DH}=$ depth hop for height; $\mathrm{LCJ}=$ lateral cone jump; $\mathrm{LBJ}=$ lateral box jump on $15-\mathrm{cm}$ box; $\mathrm{LHJ}=$ lateral hurdle jump (consecutive), $45^{\circ} \mathrm{CJ}=\mathrm{zig}-\mathrm{zag}$ cone jump; LBBJ = lateral box broad jump; MDBJ = multidirectional box jump on and off 15-cm box; DJMD = depth jump of $30 \mathrm{~cm}$ into lateral jump; LSKJ = lateral ski jump; $\mathrm{LCH}=$ lateral cone hop; LBSh = lateral box shuffle; LLH = lateral ladder hop (consecutive); $45^{\circ} \mathrm{CH}=$ zig-zag cone hop for distance (consecutive); $45^{\circ} \mathrm{CHSL}=$ zig-zag cone hop for height stick landing; MDLH = multidirectional ladder hop. $\dagger p<0.05$ indicates between-group differences for trained and untrained subjects.

\section{Practical Applications}

This study verifies that the exercises applied in the OPTIMA-Ex impact program are progressive and high intensity in nature. Our findings also demonstrate that trained subjects impart much higher magnitude loads during impact-loading exercise than those who are untrained in impact exercises; this difference between populations is an important consideration when devising an osteogenic exercise program. Moreover, these findings provide a valuable empirical reference data for magnitudes and rates of loading for a wide variety of impact exercises to facilitate evidence-based osteogenic exercise prescription.

\section{Acknowledgments}

The authors declare that there is no conflict of interest in preparing this article. The authors acknowledge and thank Haley Lewis, Cassandra Miller, Edwin Hsiao, and Jonathan Shum for their assistance during data collection and data processing for this project. This research is part of an unfunded $\mathrm{PhD}$ project, which received no specific grant from any funding agency in the public, commercial, or not-for-profit sectors. The $\mathrm{PhD}$ candidate was supported by an Australian Government Research Training Program Scholarship and Griffith University Higher Degree Research student funds. The results of this study do not constitute endorsement of the product by the authors or the NSCA. 


\section{References}

1. Abrams SA. Normal acquisition and loss of bone mass. Horm Res 60(Suppl 3): 71-76, 2003.

2. Bassey EJ, Ramsdale SJ. Increase in femoral bone density in young women following high-impact exercise. Osteoporos Int 4: 72-75, 1994.

3. Bassey EJ, Rothwell MC, Littlewood JJ, Pye DW. Pre- and postmenopausal women have different bone mineral density responses to the same high-impact exercise. J Bone Miner Res 13: 1805-1813, 1998.

4. Beck BR, Daly RM, Singh MA, Taaffe DR. Exercise and Sports Science Australia (ESSA) position statement on exercise prescription for the prevention and management of osteoporosis. J Sci Med Sport 20(5):438-445, 2016.

5. Bobbert MF, Huijing PA, van Ingen Schenau GJ. Drop jumping. II. The influence of dropping height on the biomechanics of drop jumping. Med Sci Sports Exerc 19: 339-346, 1987.

6. Burr DB, Robling AG, Turner CH. Effects of biomechanical stress on bones in animals. Bone 30: 781-786, 2002.

7. Cheng S, Sipilä S, Taaffe DR, Puolakka J, Suominen H. Change in bone mass distribution induced by hormone replacement therapy and high-impact physical exercise in post-menopausal women. Bone 31: 126-135, 2002.

8. Cummings SR, Melton LJ. Epidemiology and outcomes of osteoporotic fractures. Lancet 359: 1761-1767, 2002.

9. Ebben WP, Fauth ML, Garceau LR, Petushek EJ. Kinetic quantification of plyometric exercise intensity. J Strength Cond Res 25: 3288-3298, 2011.

10. Ebeling PR, Daly RM, Kerr DA, Kimlin MG. Building healthy bones throughout life: An evidence-informed strategy to prevent osteoporosis in Australia. Med J Aust 199: S1, 2013.

11. Heinonen A, Kannus P, Sievänen H, et al. Randomised controlled trial of effect of high-impact exercise on selected risk factors for osteoporotic fractures. Lancet 348: 1343-1347, 1996.

12. Jarvis MM, Graham-Smith P, Comfort P. A methodological approach to quantifying plyometric intensity. J Strength Cond Res 30: 2522-2532, 2016.

13. Jensen RL, Ebben WP. Quantifying plyometric intensity via rate of force development, knee joint, and ground reaction forces. J Strength Cond Res 21: 763-767, 2007.

14. Kanis JA, Melton LJ III, Christiansen C, Johnston CC, Khaltaev N. The diagnosis of osteoporosis. J Bone Miner Res 9: 1137-1141, 1994.

15. Kato T, Terashima T, Yamashita T, Hatanaka Y, Honda A, Umemura Y. Effect of low-repetition jump training on bone mineral density in young women. J Appl Physiol 100: 839-843, 2006.

16. Lambert C, Beck BR, Harding AT, Watson SL, and Weeks BK. A protocol for a randomised controlled trial of the bone response to impact loading or resistance training in young women with lower than average bone mass: the OPTIMA-Ex trial. BMJ open 7: e016983, 2017.

17. Martyn-St James M, Carroll S. Effects of different impact exercise modalities on bone mineral density in premenopausal women: A metaanalysis. J Bone Miner Metab 28: 251-267, 2010.
18. McNitt-Gray JL. Kinetics of the lower extremities during drop landings from three heights. J Biomechanics 26: 1037-1046, 1993.

19. McNitt-Gray JL. Kinematics and impulse characteristics of drop landings from 3 heights. Int J Sport Biomech 7: 201-224, 1991.

20. Peng HT. Changes in biomechanical properties during drop jumps of incremental height. J Strength Cond Res 25: 2510-2518, 2011.

21. Rubin C, Turner AS, Mallinckrodt C, Jerome C, McLeod K, Bain S. Mechanical strain, induced noninvasively in the high-frequency domain, is anabolic to cancellous bone, but not cortical bone. Bone 30: 445-452, 2002.

22. Rubin CT, Lanyon LE. Regulation of bone mass by mechanical strain magnitude. Calcif Tissue Int 37: 411-417, 1985

23. Saville PD. Osteoporosis: Disease or senescence? Lancet 1: 535, 1968.

24. Schmitt NM, Schmitt J, Dören M. The role of physical activity in the prevention of osteoporosis in postmenopausal women-An update. Maturitas 63: 34-38, 2009.

25. Seegmiller JG, McCaw ST. Ground reaction forces among gymnasts and recreational athletes in drop landings. J Athl Train 38: 311-314, 2003.

26. Self BP, Paine D. Ankle biomechanics during four landing techniques. Med Sci Sports Exerc 33: 1338-1344, 2001.

27. Sugiyama T, Yamaguchi A, Kawai S. Effects of skeletal loading on bone mass and compensation mechanism in bone: A new insight into the "mechanostat" theory. J Bone Miner Metab 20: 196-200, 2002.

28. Turner CH, Owan I, Takano Y. Mechanotransduction in bone: Role of strain rate. Am J Physiol 269: E438-E442, 1995.

29. Turner $\mathrm{CH}$, Robling AG. Designing exercise regimens to increase bone strength. Exerc Sport Sci Rev 31: 45-50, 2003.

30. Vainionpää A, Korpelainen R, Vihriälä E, Rinta-Paavola A, Leppäluoto J, Jämsä T. Intensity of exercise is associated with bone density change in premenopausal women. Osteoporos Int 17: 455-463, 2006.

31. Wallace BJ, Kernozek TW, White JM, et al. Quantification of vertical ground reaction forces of popular bilateral plyometric exercises. J Strength Cond Res 24: 207-212, 2010.

32. Wang LI, Peng HT. Biomechanical comparisons of single- and doublelegged drop jumps with changes in drop height. Int J Sports Med 35: 522-527, 2014.

33. Weeks BK and Beck BR. The BPAQ: a bone-specific physical activity assessment instrument. Osteoporos Int 19: 1567-1577, 2008.

34. Witzke KA, Snow CM. Effects of plyometric jump training on bone mass in adolescent girls. Med Sci Sports Exerc 32: 1051-1057, 2000.

35. World Health Organisation. Physical Status: The Use and Interpretation of Anthropometry: Report of the WHO Expert Committee. WHO Technical Report Series 854. Geneva, Switzerland: World Health Organisation, 1995.

36. Zhao R, Zhao M, Zhang L. Efficiency of jumping exercise in improving bone mineral density among premenopausal women: A meta-analysis. Sports Med 44: 1393-1402, 2014. 